\title{
Um Programa de Cálculo do Modelo de Hückel em Microcomputador
}

\section{Introdução}

Em Mecânica Quântica poucos sistemas são passíveis de resolução exacta havendo que recorrer, na maior parte dos casos, a métodos aproximados.

$\mathrm{O}$ modelo de Hückel foi introduzido nos fins da década de 20 e permitiu a interpretação de muitas propriedades das moléculas de hidrocarbonetos conjugados. Ele é ainda hoje muito útil, nomeadamente para a apreensão de noçð̃es cruciais em Química Quântica, quer de estrutura quer de reactividade molecular.

O objectivo deste trabalho é mostrar como o cálculo pelo método de Hückel pode ser realizado num microcomputador permitindo uma cuidada análise e interpretação dos resultados para moléculas relativamente grandes.

Este programa de cálculo foi preparado a pensar em dois tipos de destinatários:

(a) Os estudantes de Química a quem permitirá concretizar algumas noções de estrutura e reactividade molecular sem exigir mais familiaridade com o microcomputador do que a de um utilizador de programas de jogos;

(b) Os químicos interessados em interpretar propriedades de hidrocarbonetos conjugados que poderão, de modo muito expedito, obter os resultados no modelo de Hückel.

Na secção 2 faz-se uma breve exposição do modelo de Hückel para fixar a notação e para recordar alguns dos conceitos que são correntemente usados. A parte mais demorada do cálculo é a diagonalização do determinante secular que, no programa, é feita pelo algoritmo de Jacobi sumariamente descrito na secção 3. Após a descrição sumária do programa (secção 4) referem-se ainda alguns processos para melhorar e tornar mais viável a utilização deste programa.

\section{Modelo de Hückel}

O modelo de Hückel foi proposto nos fins da década de vinte por E. Hückel para o estudo da estrutura electrónica de hidrocarbonetos conjugados.

Uma das simplificaçðes cruciais usadas neste modelo é o estudo dos electrōes $\pi$ sem qualquer referência a electrōes $\sigma$.

Esta separabilidade resulta da simetria de reflexão das moléculas planas; os electrőes $\pi$ considerados por Hückel estão associados a funçð̃es de estado antissimétricas com respeito à reflexão no plano, funções que são formadas por combicação linear de orbitais atómicas $\mathrm{pz}$ dos átomos de carbono. As orbitais moleculares $\phi_{\mathrm{i}}$ são assim escritas

$\phi_{\mathrm{i}}=\sum_{\mathrm{i}=\mathrm{i}}^{\mathrm{n}} \mathrm{C}_{\mathrm{ik}} \chi_{\mathrm{k}}$ onde $\chi_{\mathrm{k}}$ é a orbital atómica $\mathrm{pz}$ centrada no átomo de carbono de índice $\mathrm{k}$. A função de estado para o sistema completo de $n$ electrð̄es escreve-se

$\Psi_{\pi}(1,2, \ldots, n)=\prod_{i=1}^{n} \phi_{i}(i)$.

A energia associada a esta função num hamiltoniano electrónico

$$
\hat{H}^{\pi}(1,2, \ldots, n)=\sum_{i=1}^{n} h^{\pi}(i)
$$

é dada pela expressão

$$
\begin{aligned}
\mathrm{E} \pi & =\sum_{\mathrm{i}=1}^{\mathrm{n}} \int \mathrm{d} \tau \phi_{\mathrm{i}}^{*} \mathrm{~h} \phi_{\mathrm{i}} \\
& =\sum_{\mathrm{i}=1}^{\mathrm{n}} \sum_{\mathrm{k}=1}^{\mathrm{n}} \sum_{1=1}^{\mathrm{n}} \mathrm{C}_{\mathrm{ik}}^{*} \mathrm{C}_{\mathrm{il}} \int \mathrm{d} \tau \chi_{\mathrm{k}} \mathrm{h} \chi_{\mathrm{l}} \\
& =\sum_{\mathrm{i}=1}^{\mathrm{n}} \sum_{\mathrm{k}=1}^{\mathrm{n}} \sum_{l=1}^{\mathrm{n}} \mathrm{C}_{\mathrm{ik}}^{*} \mathrm{C}_{\mathrm{il}} \mathrm{h}_{\mathrm{kl}}
\end{aligned}
$$

Os integrais $\mathrm{h} \pi_{\mathrm{kl}}$ nunca são calculados, sendo antes identificados com os parâmetros empíricos

$\mathrm{k}_{\mathrm{kl}}^{\pi}=\alpha$ se $\mathrm{k}=1$ se referir a um átomo de carbono,

$\mathrm{h}_{\mathrm{kl}}=\beta$ se $\mathrm{k} \neq 1$ se referirem a átomos de carbono ligados $\mathrm{e}$

$\mathrm{h} \pi_{\mathrm{k}}=0$ se $\mathrm{k} \neq \mathrm{l}$ se referirem a átomos de carbono não ligados.

Valores diferentes dos parâmetros são adoptados quando existam átomos diferentes do carbono (oxigénio ou azoto, no programa aqui apresentado). Se o átomo k for um heteroátomo $\mathrm{X}(\mathrm{X}=\mathrm{O}$ ou $\mathrm{N})$,

$$
\begin{aligned}
& \mathrm{h}_{\mathrm{kl}}^{\pi_{\mathrm{l}}}=\alpha+\mathrm{h}_{\mathrm{x}} \beta \text { se } \mathrm{k}=1 \\
& \mathrm{~h}_{\mathrm{kl}}^{\pi_{\mathrm{l}}}=\mathrm{k}_{\mathrm{l}-\mathrm{x}} \beta \text { se } \mathrm{k} \neq \mathrm{l} \text { e } 1 \text { for um átomo de carbono. }
\end{aligned}
$$

em que $h_{x}$ e $k_{1-x}$ são parâmetros empíricos característicos do heteroátomo.

Para heteroátomos com orbitais d (caso do enxôfre), o tratamento é ligeiramente diferente do anterior. Foram também propostas modificaçð̃es do modelo base em que carbonos substituídos (por exemplo por metilo, $-\mathrm{C}-\mathrm{CH}_{3}$ ) são tratados como se fossem heteroátomos com valores característicos de $h_{x}$ e $k_{1-x}$.

\footnotetext{
a Departamento de Química,

Faculdade de Ciências, Universidade do Porto 4000 Porto
} 
O objectivo primeiro do cálculo de Hückel é a resolução do sistema de equaçøes

$$
\sum_{\mathrm{l}=1}^{\mathrm{n}} \mathrm{C}_{\mathrm{il}}\left(\mathrm{h}_{\mathrm{kl}}^{\pi}-\epsilon_{\mathrm{i}}\right)=0 \quad(\mathrm{k}=1,2, \ldots, \mathrm{n})
$$

com $\mathrm{h}$ dados por (2.5) ou por (2.6) acima. Os valores próprios da matriz dos coeficientes $\mathrm{h}_{\mathrm{k} 1}^{\pi}$ são as energias das orbitais, $\epsilon_{\mathrm{i}}$, relacionadas com os coeficientes por

$$
\epsilon_{\mathrm{i}}=\sum_{\mathrm{k}=1}^{\mathrm{n}} \mathrm{C}_{\mathrm{ik}}^{2} \alpha_{\mathrm{k}}+\sum_{\mathrm{k}=1}^{\mathrm{n}} \sum_{1=1}^{\mathrm{n}} \mathrm{C}_{\mathrm{ik}}^{*} \mathrm{C}_{\mathrm{il}} \beta_{\mathrm{kl}} .
$$

A energia total do sistema electrónico da molécula é

$$
\mathrm{E}_{\pi}=\sum_{\mathrm{i}=1}^{\mathrm{n}} \mathrm{N}_{\mathrm{i}} \epsilon_{\mathrm{i}}
$$

onde $\mathrm{N}_{\mathrm{i}}$ (igual a 0,1 , ou 2) é o número de electrōes na orbital molecular $\phi_{i}$.

As quantidades $C_{\mathrm{ik}}^{2}$ e $C_{\mathrm{ik}}^{*} \mathrm{C}_{\mathrm{il}}$ que surgem na equação (2.8) acima dão uma medida da distribuição da carga electrónica do sistema $\pi$. Define-se

(i) a carga electrónica total no átomo $\mathrm{k}$

$$
\mathrm{q}_{\mathrm{k}}=\sum_{\mathrm{i}=1}^{\mathrm{n}} \mathrm{N}_{\mathrm{i}} \mathrm{C}_{\mathrm{ik}}^{2}
$$

(ii) ordem de ligação entre os átomos $\mathrm{k}$ e 1

$$
\mathrm{p}_{\mathrm{kl}}=\sum_{\mathrm{i}=1}^{\mathrm{n}} \mathrm{N}_{\mathrm{i}} \mathrm{C}_{\mathrm{ik}}^{*} \mathrm{C}_{\mathrm{il}}
$$

Outras quantidades calculáveis na teoria de Hückel têm sido correlacionadas com a reactividade molecular.

A valência livre $\left(F_{k}\right)$ dá uma medida da capacidade residual dos átomos de carbono de radicais para estabelecer ligaçð̃es e é calculada como a diferença entre a capacidade máxima de ligação $\pi$ de um átomo de carbono e o seu valor no sistema considerado,

$$
\mathrm{F}_{\mathrm{k}}=\sqrt{3}-\sum_{\ell} \mathrm{p}_{\mathrm{k} \ell}
$$

onde o somatório se refere apenas a átomos $\ell$ vizinhos de $\mathrm{k}$.

A polarizibilidade atómica, $\pi_{\mathrm{k},}$ reflecte a mudança que ocorre (em primeira ordem de teoria de perturbaçōes) na densidade de carga $\pi$ de um átomo $\mathrm{k}$ em consequência de uma alteração unitária da densidade de carga $\pi$ do átomo $\ell$ e é dada por

$$
\begin{aligned}
\pi_{\mathrm{k} \ell} & =\frac{\partial \mathrm{q}_{\mathrm{k}}}{\partial \alpha_{\ell}} \\
& =4 \sum_{\mathrm{i}} \sum_{\mathrm{j}} \frac{\mathrm{C}_{\mathrm{ik}}^{2} \mathrm{C}_{\mathrm{j} \ell}^{2}}{\epsilon_{\mathrm{i}}-\epsilon_{\mathrm{j}}}
\end{aligned}
$$

onde os somatórios incluem apenas orbitais ocupadas e desocupadas com energia diferente, $\epsilon_{\mathrm{i}} \neq \epsilon_{\mathrm{j}}$.

\section{Resolução das equações seculares.} Algoritmo de Jacobi

Nesta seç̧ão expð̃e-se de uma forma abreviada o método de Jacobi para a diagonalização de matrizes. Usa-se a notação matricial para evitar expressð̋es aparentemente complexas pela ocorrência de índices repetidos.
A leitura desta secção não é essencial para a compreensão das seguintes nem para a utilização do programa. A principal dificuldade do modelo de Hückel reside na resolução das equaçס̃es seculares apresentadas em (2.7)

$$
\sum_{\ell} \mathrm{C}_{\mathrm{i} \ell}\left(\mathrm{h}_{\mathrm{k} \ell}^{\pi}-\epsilon_{\mathrm{j}}\right)=0
$$

Traduzindo ou representando essas equaçס̃es na forma matricial, ter-se-á

$$
\mathbf{H} \mathbf{C}=\mathbf{C} \mathbf{E}
$$

em que $\mathbf{H}$ e $\mathbf{C}$ são matrizes quadradas $(n \times n)$ com elementos $\mathrm{h}_{\mathrm{k} \ell}^{\pi}$ e $\mathrm{C}_{\mathrm{il}}$, e $\mathbf{E}$ é a matriz quadrada diagonal $(\mathrm{n} \times \mathrm{n})$ cujos valores próprios $\epsilon_{\mathrm{i}}$ são as energias das orbitas moleculares (OM), $\phi_{\mathrm{i}}$.

Por outro lado, como as $\mathrm{OM}, \phi_{\mathrm{i}}$, devem ser ortonormais, a matriz dos coeficientes $\mathrm{C}_{\mathrm{i} \ell}$ terá de satisfazer a relação

$$
\mathbf{C}^{+} \mathbf{C}=\mathbf{1}
$$

onde a notação + indica conjugação de Hermite (ou transposição se $\mathbf{C}$ for real) e a matriz $\mathbf{1}$ é a matriz unidade. A matriz C diz-se ortogonal, por satisfazer a relação anterior.

Existem vários métodos numéricos de resolução do problema matricial de valores próprios expresso em (3.2), sendo talvez, o mais usado em Química Quântica, o método de Jacobi. Este é um método bem estabelecido, fácil de implementar num programa de cálculo e fornece simultaneamente os valores próprios $\epsilon$ e correspondentes vectores próprios $\mathbf{C}$.

Repare-se que se se multiplicar a equação (3.2) por $\mathbf{C}^{+}$ $\mathrm{e}$ atendendo a que $\mathbf{C}$ é uma matriz ortogonal, obter-se-á:

$$
\mathrm{C}^{+} \mathrm{H} \mathrm{C}=\mathrm{C}^{+} \mathrm{CE}=\mathbf{1} \mathrm{E}=\mathrm{E}
$$

Reduz-se então o problema à determinação de uma matriz $\mathbf{C}$ ortogonal, constituída pelos vectores proprios $\mathrm{C}_{\mathrm{i}}$, que por uma transformação de similaridade do tipo (3.4) diagonalize a matriz simétrica de Hückel $\mathbf{H}$. Esta matriz C é, no método de Jacobi, obtida por um processo de aproximaçð̌es sucessivas, em que a matriz $\mathbf{H}$ vai sendo progressivamente modificada $\left(\mathbf{H}^{(1)} \rightarrow \mathbf{H}^{(2)} \rightarrow \mathbf{H}^{(3)} \rightarrow \mathbf{H}^{(4)} \rightarrow \ldots \ldots \rightarrow \mathbf{H}^{(\mathrm{n})}\right)$

$$
\begin{aligned}
& \mathbf{O}_{1}^{+} \mathbf{H O}_{1}=\mathbf{H}^{(1)} \\
& \mathbf{O}_{2}^{+} \mathbf{H}^{(1)} \mathbf{O}_{2}=\mathbf{H}^{(2)}, \text { etc., }
\end{aligned}
$$

até se achar uma matriz $\mathbf{H}^{(\mathrm{n})}$ aproximadamente diagonal,

$$
\mathbf{O}_{\mathrm{n}}^{+} \mathbf{H}^{(\mathrm{n}-1)} \mathbf{O}_{\mathrm{n}}=\mathbf{H}^{(\mathrm{n})}=\mathbf{E}
$$

$\mathrm{O}$ que é equivalente a

$$
\mathbf{O}_{\mathrm{n}}^{+} \mathbf{O}_{\mathrm{n}-1}^{+} \ldots \mathbf{O}_{\mathrm{i}}^{+} \mathbf{H} \mathbf{O}_{1} \ldots \mathbf{O}_{\mathrm{n}-1} \mathbf{O}_{\mathrm{n}}=\mathbf{E}
$$

sendo a matriz $\mathbf{C}$ dos coeficientes, dada então pelo produtório:

$$
\mathbf{C}=\underset{\mathrm{p}=1}{\pi_{\mathrm{n}}^{\mathrm{n}}} \mathbf{O}_{\mathrm{p}}
$$


As matrizes ortogonais $\mathbf{O}_{\mathrm{p}}$ usadas, são matrizes de rotação de forma semelhante à apresentada seguidamente para $\mathbf{O}_{1}$

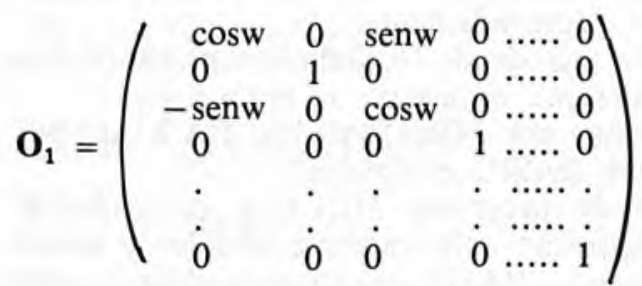

A matriz $\mathbf{O}_{1}$, neste caso, realiza sobre $\mathbf{H}$ uma rotação de ângulo $w$ no plano $(1,3)$. Os elementos $h_{13}$ e $h_{31}$ podem ser anulados por escolha conveniente do valor $w$. Cada matriz $\mathbf{O}_{\mathrm{p}}$ é constituída de modo a anular o elemento $\mathrm{h}_{\mathrm{k} \ell}^{(\mathrm{p})}$, da matriz $\mathbf{H}^{(\mathrm{p})}$, de maior valor absoluto. Este processo cíclico considera-se terminado quando os elementos não diagonais da matriz produzida na transformação $n$ não forem superiores a um dado valor predeterminado (geralmente $10^{-8}$ ou $10^{-10}$ ).

Genericamente, os elementos da matriz $\mathbf{H}^{(\mathrm{p}+1)}$ obtidos na rotação $\mathrm{p}$, tem a forma:

$$
\begin{aligned}
& \mathrm{h}_{\mathrm{km}}^{(\mathrm{p}+1)}(\mathrm{m} \neq \mathrm{k}, \ell)=\mathrm{h}_{\mathrm{km}}^{(\mathrm{p})} \operatorname{cosw}-\mathrm{h}_{\mathrm{m}}^{(\mathrm{p})} \text { senw }= \\
& =\mathrm{h}_{\mathrm{mk}}^{(\mathrm{p}+1)} \\
& \mathrm{h}_{\mathrm{gm}}^{(\mathrm{p}+1)}(\mathrm{m} \neq \mathrm{k}, \ell)=\mathrm{h}_{\mathrm{km}}^{(\mathrm{p})} \operatorname{senw}-\mathrm{h}_{\mathrm{m}}^{(\mathrm{p})} \cos \mathrm{w}= \\
& =\mathrm{h}_{\mathrm{m} \ell}^{(\mathrm{p}+1)} \\
& \mathrm{h}_{\mathrm{kk}}^{(\mathrm{p}+1)}=\frac{1}{2}\left(\mathrm{~h}_{\mathrm{kk}}^{(\mathrm{p})}+\mathrm{h}_{\mathscr{Q}}^{(\mathrm{p})}\right)+ \\
& +\frac{1}{2}\left(h_{k k}^{(p)}-h_{(\ell)}^{(p)} \cos 2 w-h(p) \operatorname{sen} 2 w\right. \\
& \mathrm{h}_{\mathscr{Q}}^{(\mathrm{p}+1)}=\frac{1}{2}\left(\mathrm{~h}_{\mathrm{kk}}^{(\mathrm{p})}+\mathrm{h}_{\mathscr{Q}}^{(\mathrm{p})}\right)- \\
& -\frac{1}{2}(\mathrm{~h}(\mathrm{p})-\mathrm{h}(\mathrm{p})) \cos 2 \mathrm{w}-\mathrm{h}(\mathrm{p}) \operatorname{sen} 2 \mathrm{w} \\
& \mathrm{h}_{\mathfrak{Q}}^{(\mathrm{p}+1)}=\mathrm{h}_{\mathfrak{Q} \mathrm{k}}^{(\mathrm{p}+1)}=0= \\
& =\frac{1}{2}\left(h_{k k}^{(p)}-h_{(p)}^{(p)}\right) \operatorname{sen} 2 w-h(p) \cos 2 w
\end{aligned}
$$

O valor w é então dado pela expressão

$$
-\left(h_{k k}^{(p)}-h_{\dddot{w}}^{(p)}\right) \operatorname{sen} 2 w+2 h_{k \ell}^{(p)} \cos 2 w=0
$$

ou seja,

$$
\operatorname{tg}(2 \mathrm{w})=2 \mathrm{~h}_{\mathrm{k} \ell}^{(\mathrm{p})} /\left(\mathrm{h}_{\mathscr{Q}}^{(\mathrm{p})}-\mathrm{h}_{\mathrm{kk}}^{(\mathrm{p})}\right)
$$

com $\mathrm{w} \in[-\pi / 4, \pi / 4]$.

Contudo, verifica-se geralmente que o número de transformaçōes necessárias à diagonalização de $\mathbf{H}$ é superior ao número de elementos não diagonais desta. De facto, não existe garantia de que um elemento $h_{k \ell}$ anulado numa dada transformação, não volte a ser tornado diferente de zero numa transformação posterior. Deste modo, o processo de Jacobi pode tornar-se muito lento.

Depois de determinadas, por este método, as matrizes C e E, rapidamente se calculam as propriedades já referidas, nomeadamente:
- As orbitais moleculares (equação 2.1)

- A energia electrónica $\pi$ total (equação 2.4)

- As densidades de carga electrónica $\pi$ (equação 2.10)

- As ordens de ligação $\pi$ (equação 2.11)

- As valências livres (equação 2.12)

- E as polarizabilidades atómicas (equação 2.13)

\section{Estrutura do programa HÜCKEL}

O programa HÜCKEL, foi escrito na linguagem de programação Applesoft/Basic e pode ser executado em microcomputadores da família Apple //.

O programa permite realizar cálculos de hidrocarbonetos conjugados constituídos por, no máximo, 30 átomos de carbono, sendo também aplicável a hidrocarbonetos com heteroátomos de azoto, oxigénio ou enxofre. Os valores dos parâmetros $\alpha_{\mathrm{k}}$ e $\beta_{\mathrm{k} \ell}$ utilizados por este programa, no caso de heteroátomos, são os recomendados por Streitwieser (1).

O programa está subdividido num programa principal e 6 subprogramas cujas designações e objectivos são os seguintes:

- Programa principal HELLO: Inicia a leitura dos dados da molécula necessários à construção da matriz de Hückel. Contém ainda um menu que permite, ao utilizador, escolher a propriedade que deseja conhecer para a molécula em estudo.

- Subprograma DADOS: Diagonaliza a matriz de Hückel utilizando o método de Jacobi. Ordena também, por ordem crescente, os elementos da matriz $\mathbf{E}$ resultante e permuta convenientemente as colunas da matriz $\mathbf{C}$ que com eles devem permanecer associados.

- Subprograma OM: Constroi todas as orbitais moleculares (ligantes, não ligantes e anti-ligantes) e determina a energia electrónica $\pi$ total do sistema.

- Subprograma CARGAS: Calcula a densidade de carga electrónica $\pi$ de cada átomo do sistema.

- Subprograma ORDENS: Determina a ordem de ligação $\pi$ para cada par de átomos do sistema.

- Subprograma VAL: Calcula as valências livres de cada átomo do sistema.

- Subprograma POL: Determina as polarizabilidades atómicas para cada par de átomos do sistema se este for de camada fechada (número de electrōes par).

$\mathrm{Na}$ figura 1 apresenta-se em esquema a estrutura do programa HÜCKEL, na qual se procura evidenciar o fluxo de controle gerado durante a sua execução.

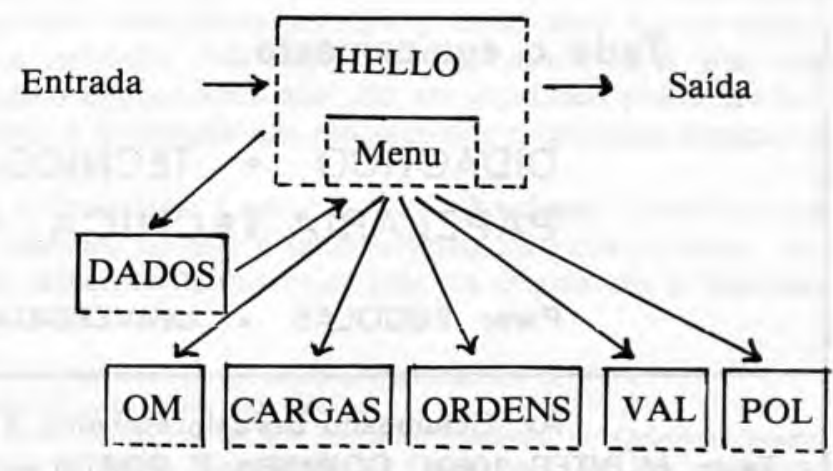

Fig. 1 - Diagrama de fluxos do programa HUCKEL 


\section{Resultados e conclusões}

O programa HÜCKEL aqui apresentado foi testado com uma série de moléculas, tendo os resultados sido verificados por comparação com resultados publicados (refs. 1,2 e 3 ).

$\mathrm{Na}$ execução do programa, a fase que consome mais tempo é a diagonalização da matriz de Hückel, sendo os resultados obtidos num Apple //e apresentados na Tabela 1.

Tabela 1 - Tempo gasto na diagonalização da matriz de Hückel para várias espécies químicas

Espécie química $n .^{\circ}$ de átomos de carbono tempo(s)

\section{Etileno}

Alilo

Butadieno

Pentadienilo

Benzeno

Heptatrienilo

Octatetraeno

Benzociclopentadienilo

Bifenilo

1-Femalenilo (*)

(*) Espécie radicalar que se pode representar sob a forma
Deve notar-se que o tempo de execução do programa, especialmente para moléculas de maior dimensão, poderá vir a ser diminuído por utilização de um algoritmo de diagonalização mais eficaz ou de uma linguagem compilável. Nomeadamente:

(a) utilização do método de Householder ou de Givens para a diagonalização da matriz de Hückel ou

(b) a programação em FORTRAN ou PASCAL ou, pelo menos, num BASIC compilável.

$\mathrm{Na}$ preparação do programa HÜCKEL pretendeu-se adoptar um algoritmo relativamente simples e numa versão da linguagem BASIC muito expandida e onde alguns utentes poderão vir a introduzir modificaçð̄es que adaptem este programa às suas necessidades específicas.

O programa HÜCKEL, escrito para microcomputadores Apple //e ou Apple $/ / \mathrm{c}$, é posto à disposição dos leitores. Para obter uma cópia do programa e manual respectivo deverá enviar aos autores um disco magnético flexível de 5"1/4 e $100 \$ 00$ para despesas de expedição.

\section{BIBLIOGRAFIA}

1-A. Streitwieser - Molecular Orbital Theory, John Wiley \& Sons, Inc. (1962).

2 - J.P. Lowe - Quantum Chemistry, Student Edition, Academic Press, Inc. (1978).

$3-$ R. Zahradnik e P. Cársky - Organic Quantum Chemistry Problems, Plenum Press (1973).

4-A.R. Gourbuy e G.A. Watson - Computational Methods for Matrix Eigenproblems, John Wiley \& Sons, Ltd. (1973).

$5-\mathrm{G}$. Beech - Fortran IV in Chemistry, An Introdution to Computer Assisted Methods, John Wiley \& Sons, Ltd. (1975).

Todo o equipamento :

nucleon

EOUIPAMENTOS DE PRECISÃO LDA.

DIDACTICO - TÉCNICO • CIENTIFICO • DESENHO PAPELARIA TECNICA • TOPOGRAFIA E GEODESIA

Para: ESCOLAS - UŃIVERSIDADES - INVESTIGAÇÃO - INDÚSTRIA

Av. Columbano Bordalo Pinheiro, 57-A - Telefs. 724748/722039-1000 LISBOA

Telex MUNTER 16691 COIMBRA E PORTO - Av. Sá da Bandeira, 62, Telef. 33778, 3000 COIMBRA 